\title{
Polarization plane rotator used as a phase stepping device in a 2-channel shearing speckle interferometer
}

\author{
Peter A.A.M. Somers ${ }^{*}$, Nandini Bhattacharya \\ Optics Research Group, Delft University of Technology \\ Lorentzweg 1, NL-2628 CJ Delft, the Netherlands
}

\begin{abstract}
A polarization phase stepping method is presented based on the use of a polarization plane rotator that establishes a relative phase shift between two counter-rotating circularly polarized beams. The phase step can be made relatively accurate, since it just depends on the accuracy with which the rotator is manufactured, and not on its orientation. The phase stepping method has been implemented in a single-camera two-channel shearing speckle interferometer, with two optical channels, and a relative phase step of $\pi / 2$ between them.
\end{abstract}

Keywords: speckle interferometry, polarization phase stepping, polarization plane rotator

\section{INTRODUCTION}

An important feature for optical measurement systems for industrial inspection is their usefulness at locations outside the laboratory. Interferometry is a powerful tool to measure very small displacements or deviations from a standard shape of an object - even down to a fraction of a nanometer - but its sensitivity can also be a serious drawback, requiring conditions that can not be met in industrial environments. In addition, many surfaces of industrial objects are diffusely reflective, causing speckles that have been considered as a noise source for a long time in interferometry, and indeed do have a negative influence in measurement systems for reflective surfaces. Later it was recognized that speckles can be considered as information carriers too, and speckle interferometry saw the light.

The standard speckle interferometer acquires a speckle interferogram that is obtained by interference between a speckle field, which can be observed when a diffusely reflecting surface is illuminated by a coherent light source and a reference beam from the same source. The reference beam generally is a planar or spherical wave. The interferometer can be made sensitive for in plane or out of plane displacements, but is also sensitive for environmental influences: any change of the optical path length difference between object speckle pattern and the reference beam is measured.

However, there is a class of speckle interferometers that has reduced sensitivity for environmental influences: the shearing speckle interferometer. In contrast to the standard speckle interferometer the shearing speckle interferometer derives its reference from the inspected object itself, by superimposing two speckle patterns that are slightly shifted with respect to each other. This creates an almost common path for object and reference beams, rendering the set-up relatively insensitive for object translations towards the interferometer. The set-up also provides reduced sensitivity for air turbulence between the object and the interferometer, and allows for more relaxed requirements with respect to the minimum coherence length of the illumination source. Besides this, the sensitivity for the displacement that has to be measured can be adjusted by changing the shearing distance, i.e. the shift between the two speckle patterns. The most important drawback of shearography when compared to standard speckle pattern interferometry is lack of modulation at positions in the interference pattern where a speckle from one pattern does not overlap with one from the other.

Deformation of the object due to applying a load will lead to local displacements that can be measured with shearography since it is differentially sensitive in the direction of the applied shear. Global displacements don't lead to differential displacements and are not measured. Phase differences caused by deformation of the object can be quantified by acquiring at least three speckle interferograms, each taken with a different instance of the reference beam. Each

*P.A.A.M.Somers@TNW.TUDelft.nl; N.Bhattacharya@TNW.TUDelft.nl; phone +31 (0)15 27 84288; fax +31 (0)15 2788105 ; http://www.optica.tn.tudelft.nl/ 
instance of the reference beam is given a certain phase shift compared to the initial phase of the reference beam. Common values of the phase step are multiples of $\pi / 2$ or $2 \pi / 3$. Phase can then be calculated at each point in the interferogram, using one of the well-known phase stepping algorithms ${ }^{1}$, and phase change due to deformation of the object can be determined by subtraction of phase distributions before and after the event that changed the status of the object.

Phase stepping can be implemented temporally or spatially. The former implies creating a change in the relative phase between object and reference beams after acquisition of the first speckle interferogram and then taking a next one. This is repeated until the required number of interferograms for the chosen algorithm is obtained. During acquisition of the set of interferograms the experimental conditions are assumed not to have altered, which may not be the case in an industrial environment, resulting in phase errors. In addition the most often used phase stepping mechanism, the piezo mirror translator that mechanically changes the length of one of the optical paths, suffers from non-linear behaviour, hysteresis and drift, causing further errors in the calculation of phase.

Other mechanical methods for temporal phase stepping use piezo driven devices that can change the length of an optical fiber, through which the reference beam is sent. Alternatively, its temperature can be changed to obtain the same result. Parallel plate or wedge based phase stepped systems, or shifting gratings can be used as well for temporal phase stepping. Polarization sensitive elements such as waveplates can be positioned in different orientations to create relative phase changes between object and reference beams ${ }^{2-4}$. All these methods are relatively slow. Electrically controlled devices such as liquid crystal wave plates, acousto-optical or electro-optical devices can be used to implement faster phase stepping devices. Also the wavelength of the laser can be changed to obtain fast temporal phase shifts.

To avoid even the smallest time delay between phase steps, phase stepping can also be implemented spatially. All interferograms are taken at the same moment in time, which eliminates errors encountered with temporal phase stepping when phase is changing during phase stepping. Basically two approaches are followed: in the first one a spatial carrier is added by tilting the uniform reference beam. Adjacent pixels now have different reference phase, and the speckle interference pattern can be processed with the Fourier method ${ }^{5}$. If phase difference between adjacent pixels is for instance $\pi / 2$ and three or more phase stepped pixels are available, phase can be calculated for the set using one of the well known phase stepping algorithms ${ }^{6}$. Speckle size is assumed to be large enough to accommodate three or more phase steps for the same speckle, which reduces the efficiency of the use of the available light. Another one-image approach is the use of a diffraction grating that projects a set of speckle patterns on a CCD, each phase stepped speckle pattern at a different position ${ }^{7}$.

Another approach for spatial phase stepping is the use of multiple optical channels, each channel with its own phase step. Speckle patterns can be projected onto a single camera ${ }^{8}$, or each pattern can have its own camera ${ }^{9}$. An advantage of the multiple channel approach when compared to systems employing a spatial carrier is the more efficient use of the available light. A major challenge for single camera systems with multiple images, or multiple camera systems is the alignment of the speckle patterns, which has to be realized with sub-pixel accuracy, in order to allow speckle size to be as small as pixel size ${ }^{10}$. A three or four channel system is also more complex than single image spatial phase stepped systems or temporal phase stepped systems. A comprehensive review of phase stepping methods is given by Dorrio ${ }^{11}$.

The speckle shearing interferometer that will be discussed in the next sections is a compromise between several requirements. Above all, the system should perform well in industrial environments, and exhibit the lowest possible sensitivity for environmental disturbances, which has led to the choice for a spatial phase stepped system. The wish to achieve the most efficient use of available light allowing relatively small illumination sources resulted in opting for a multiple channel system. The system has been kept relatively simple by reducing the number of channels to two, instead of three or four that are required to calculate phase. Since our interest was only the determination of phase difference and not phase, a two-bucket algorithm could be selected, and system hardware could be limited to only two phase stepped speckle interferograms.

\section{ALGORITHMS}

For reasons of simplicity a two-bucket phase stepping algorithm has been chosen, combined with temporal phase unwrapping $^{12}$, which produces real-time unwrapped phase difference maps that can be immediately interpreted. Four 
equations are involved, obtained by acquiring two pairs of phase stepped speckle interferograms: one pair taken before the event that changes the object, and one after it. There are four unknowns at each point of the interferogram: the amplitude of object and reference beams, their relative phase, and the unknown phase change. In principle the four equations are sufficient to resolve all variables, but our main interest is phase change, which can be calculated with some restrictions ${ }^{13}$. The four equations, each expressing the measured intensity at a given point $x, y$ in the interferogram in the four unknowns are:

$$
\begin{aligned}
& I_{0}(t)=I_{o}+I_{r}+2 \sqrt{I_{o} I_{r}} \cos (\varphi), \\
& I_{\pi / 2}(t)=I_{o}+I_{r}+2 \sqrt{I_{o} I_{r}} \cos (\varphi-\pi / 2), \\
& I_{0}(t+\Delta t)=I_{o}+I_{r}+2 \sqrt{I_{o} I_{r}} \cos (\varphi+\Delta \varphi), \\
& I_{\pi / 2}(t+\Delta t)=I_{o}+I_{r}+2 \sqrt{I_{o} I_{r}} \cos (\varphi+\Delta \varphi-\pi / 2),
\end{aligned}
$$

where $I_{o}$ and $I_{r}$ are the intensities of the object and reference beams respectively, $\varphi$ is the initial phase difference between the two and $\Delta \varphi$ is phase difference caused by the event. Indexes 0 and $\pi / 2$ for the intensities denote phase steps 0 and $\pi / 2, t$ and $t+\Delta t$ denote moments in time: $t$ is used for the intensity before the event, $t+\Delta t$ for the status after. These equations can be written as:

$$
\begin{aligned}
& I_{0}(t)=I_{B}+I_{M} \cos (\varphi) \\
& I_{\pi / 2}(t)=I_{B}+I_{M} \cos (\varphi-\pi / 2) \\
& I_{0}(t+\Delta t)=I_{B}+I_{M} \cos (\varphi+\Delta \varphi) \\
& I_{\pi / 2}(t+\Delta t)=I_{B}+I_{M} \cos (\varphi+\Delta \varphi-\pi / 2)
\end{aligned}
$$

where $I_{B}$ is background intensity and $I_{M}$ is modulation intensity.

Assuming that background and modulation intensity have not changed due to the event, Eqs. (5-8) yield:

$$
\Delta \varphi=\pi / 2+2 \arctan \left[\frac{I_{0}(t)-I_{\pi / 2}(t+\Delta t)}{I_{\pi / 2}(t)-I_{0}(t+\Delta t)}\right]
$$

This expression is only valid ${ }^{13}$ if the average phase at the specific point is not equal to $\pi / 4$ or $5 \pi / 4$. Invalid pixels, and pixels that are unreliable due to low modulation can be replaced by spatial filtering.

An event that may cause phase changes that are larger than $+/-\pi$ can be handled by dividing the measurement interval into a number of intervals for each of which the phase change is smaller than $+/-\pi$. Phase changes are calculated for each interval using Eq. (9), spatially filtered, and accumulated, eliminating the need for spatial phase unwrapping after completion of the test. Unwrapped results can be displayed in real time during the test. The procedure is known as temporal phase unwrapping ${ }^{12}$.

\section{POLARIZATION PHASE STEPPING}

In a spatial phase stepping interferometer the phase stepping device can be built using polarization sensitive elements such as quarter wave plates, half wave plates or more complex devices such as Savart elements ${ }^{14}$ or custom made components ${ }^{9}$. Object and reference beams with different polarization states travel through a common path, in which a birefringent element can be placed to introduce polarization dependent retardation of the beams. If two beams with mutual orthogonal linear polarization directions travel through the same optical path and a retarder wave plate is placed in the path with its slow axis coinciding with the polarization direction of one of the beams, that beam will be retarded relative to the other one. Phase difference is $\pi$ for a half wave plate. Similarly a quarter wave plate introduces a phase difference of $\pi / 2$. The wave plates must be positioned carefully; deviations from the ideal orientation will cause errors. For a multiple channel system each optical branch except one must have an appropriate retarder plate; for a two-channel 
system one retarder plate is placed into one of the optical branches. The phase stepping section must be preceded by a module that conditions incoming light and delivers two mutual orthogonal polarization states travelling through a single optical path. The reference beam may be horizontally polarized and the object beam vertically, or vice versa. The single optical path is split into two optical branches, and in one of them the retarder plate is positioned. Analyzers in each of the branches, oriented at $45^{\circ}$, will cause the beams to interfere at a CCD camera, placed behind each of the analyzers, as shown in Fig. 1.

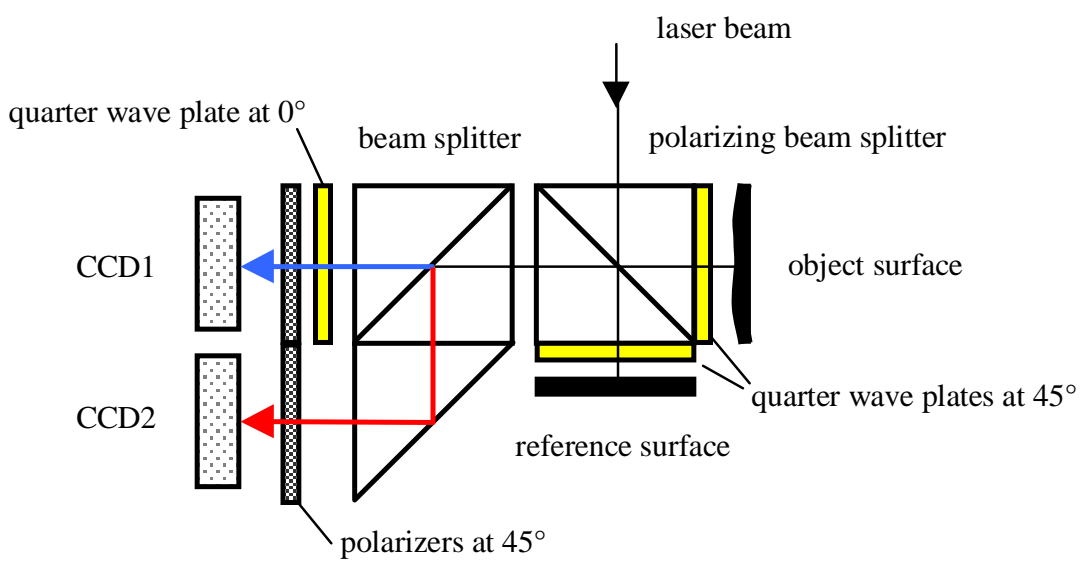

Figure 1: Polarization phase stepping for mutual orthogonal linearly polarized beams with a quarter wave plate.

Another solution is to make both beams circular, one left-handed, the other right-handed circular and achieving phase stepping by placing both analysers at different orientations. Rotation of an analyzer over an angle of $\alpha$ creates a phase step for the interference pattern of $2 \alpha$, since rotation of the analyzer influences phase for right- and left-handed light simultaneously and causes phase changes in opposite directions. For a phase step of $\pi / 2$, the relative orientation of the two analyzers should be $\pi / 4$. One analyzer could be oriented at $\pi / 8$ relative to the horizontal plane, and the other one at $-\pi / 8$. The set-up is shown in Fig. 2.

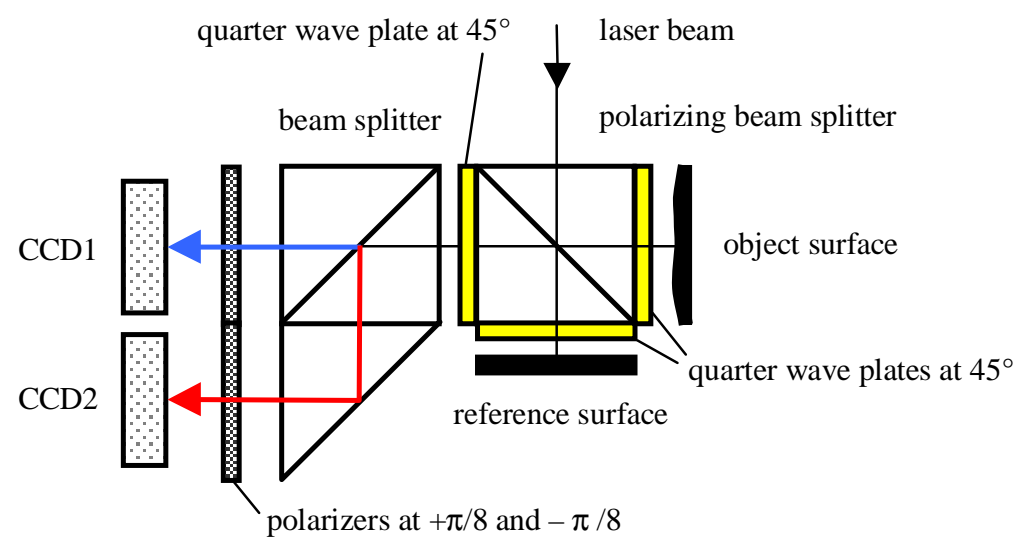

Figure 2: Polarization phase stepping for circularly polarized beams with opposite direction, with two analyzers, positioned at $+\pi / 8$ and $-\pi / 8$.

A third solution is based again on the use of circular light, and two analyzers, both oriented horizontally. One of the optical branches now requires rotation of the circular beams over an angle of $\alpha=\pi / 4$ in order to achieve a phase step of $\pi / 2$. This can be done by a half wave plate oriented at $\pi / 8$ relative to the horizontal plane, or by a polarization plane rotator, manufactured for a rotation angle of $\pi / 4$. The latter option is preferred since the orientation of the half wave plate is critical ${ }^{4}$, and the orientation of the rotator is not. The transmission axes of both analyzers are now zero. A two-camera set-up is shown in Fig. 3. 


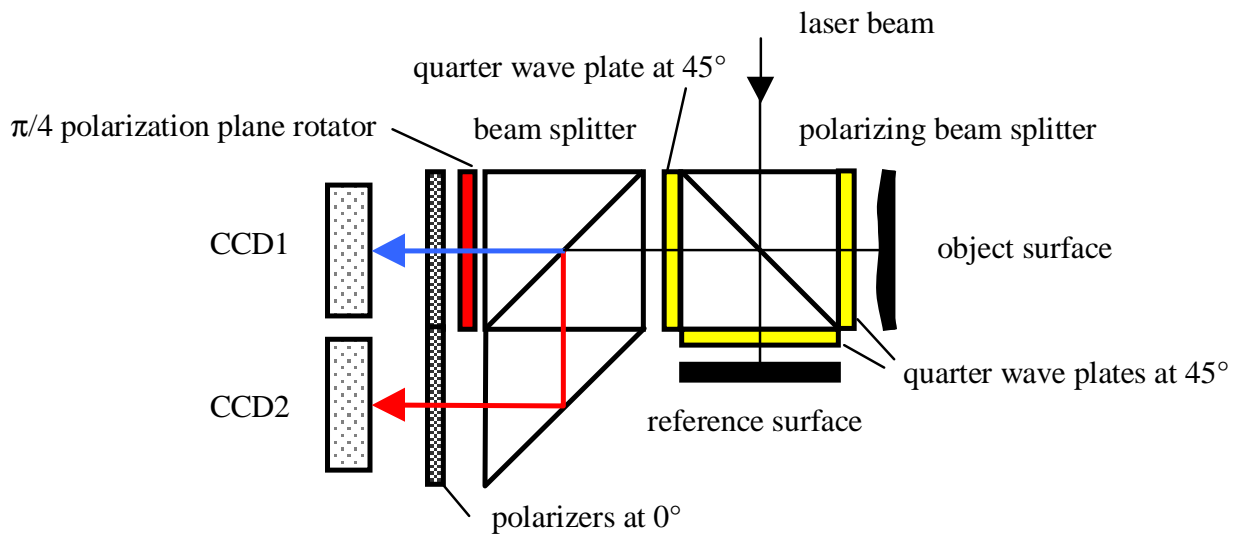

Figure 3: Polarization phase stepping of circularly polarized beams with opposite polarization direction, with a polarization plane rotator and two analyzers, positioned at $0^{\circ}$.

A polarization sensitive combiner could be used to combine both images and project them side by side onto a single CCD camera. Horizontal orientation of the transmission axes of both analyzers is convenient in that case, so the solution presented in Fig. 3 is the most appropriate.

The properties of the phase stepping system, in particular the role of the polarization plane rotator can be described mathematically by using the Jones calculus ${ }^{15,16}$. Polarized light can be characterized by its complex amplitude, represented by the matrix expression:

$$
E_{0}=\left[\begin{array}{c}
E_{0 x} e^{i \varphi_{x}} \\
E_{0 y} e^{i \varphi_{y}}
\end{array}\right],
$$

where $E_{0 x}$ and $E_{0 y}$ represent the field amplitudes in $x$ - and $y$-direction, and $\varphi_{x}$ and $\varphi_{y}$ represent phase for $x$ - and $y$ directions respectively.

If $E_{x}$ leads $E_{y}$ by $\pi / 2$ we have left-circularly polarized light. If $\varphi_{x}$ equals to zero, and after normalizing, Eq. (10) can be written as:

$$
E_{0}=\frac{1}{\sqrt{2}}\left[\begin{array}{l}
1 \\
i
\end{array}\right]
$$

Similarly right-circularly polarized light can be described by:

$$
E_{0}=\frac{1}{\sqrt{2}}\left[\begin{array}{l}
1 \\
-i
\end{array}\right]
$$

A horizontal polarizer can be represented by the following Jones matrix:

$$
M_{H P}=\left[\begin{array}{ll}
1 & 0 \\
0 & 0
\end{array}\right]
$$

Passing left-handed circular light through a horizontal polarizer can then be described by the matrix operation:

$$
V_{1}=M_{H P} V_{L C}
$$


where $V_{L C}$ represents the Jones vector for left-circularly polarized light. Similarly, for right-hand circular light passing through a horizontal polarizer follows:

$$
V_{2}=M_{H P} V_{R C}
$$

The results of the matrix operations are identical:

$$
V_{1}=\frac{1}{\sqrt{2}}\left[\begin{array}{l}
1 \\
0
\end{array}\right]
$$

and

$$
V_{2}=\frac{1}{\sqrt{2}}\left[\begin{array}{l}
1 \\
0
\end{array}\right]
$$

Vectors $V_{1}$ and $V_{2}$ represent in-phase horizontally polarized light.

The Jones matrix $\mathrm{R}$ for a polarizing plane rotator that rotates the polarization direction over an angle $\beta$ is identical to the rotation matrix ${ }^{16}$ :

$$
R=\left[\begin{array}{cc}
\cos \beta & -\sin \beta \\
\sin \beta & \cos \beta
\end{array}\right]
$$

Operating the rotation matrix $\mathrm{R}$ on the Jones vector for left-handed circular light yields:

$$
V_{\text {LCRot }}=\frac{1}{\sqrt{2}}\left[\begin{array}{cc}
\cos \beta & -\sin \beta \\
\sin \beta & \cos \beta
\end{array}\right]\left[\begin{array}{l}
1 \\
i
\end{array}\right]=\frac{1}{\sqrt{2}}\left[\begin{array}{l}
\cos \beta-i \sin \beta \\
\sin \beta+i \cos \beta
\end{array}\right]
$$

which is equivalent to:

$$
V_{\text {LCRot }}=\frac{1}{\sqrt{2}}\left[\begin{array}{l}
e^{-i \beta} \\
i e^{-i \beta}
\end{array}\right]=\frac{e^{-i \beta}}{\sqrt{2}}\left[\begin{array}{l}
1 \\
i
\end{array}\right]
$$

Similarly operating the matrix R on the Jones vector for right-handed circular light yields:

$$
V_{\text {RCRot }}=\frac{1}{\sqrt{2}}\left[\begin{array}{cc}
\cos \beta & -\sin \beta \\
\sin \beta & \cos \beta
\end{array}\right]\left[\begin{array}{l}
1 \\
-i
\end{array}\right]=\frac{1}{\sqrt{2}}\left[\begin{array}{c}
\cos \beta+i \sin \beta \\
\sin \beta-i \cos \beta
\end{array}\right]
$$

which is equivalent to:

$$
V_{R C R o t}=\frac{1}{\sqrt{2}}\left[\begin{array}{l}
e^{i \beta} \\
-i e^{i \beta}
\end{array}\right]=\frac{e^{i \beta}}{\sqrt{2}}\left[\begin{array}{l}
1 \\
-i
\end{array}\right]
$$

Each of the circular beams has undergone a phase shift of $\beta$ with opposite sign for left- and right-handed circular light, resulting in a total relative phase shift of $2 \beta$. These phase shifted beams travel through the polarizer, yielding $V_{l R o t}$ and $V_{2 R o t}$, which can be described by the matrix operation:

$$
V_{\text {lRot }}=M_{H P} V_{L C R o t},
$$


where $V_{L C R o t}$ represents the Jones vector for left-circularly polarized light after passing the rotator. Similarly, for righthanded circular light passing through a horizontal polarizer follows:

$$
V_{2 R o t}=M_{H P} V_{R C R o t}
$$

Substituting Eqs. (13), (19) and (21) for a horizontal polarizer operating on left- and right-handed circular light:

$$
V_{1 R o t}=\frac{1}{\sqrt{2}}\left[\begin{array}{ll}
1 & 0 \\
0 & 0
\end{array}\right]\left[\begin{array}{c}
\cos \beta-i \sin \beta \\
\sin \beta+i \cos \beta
\end{array}\right]
$$

and

$$
V_{2 R o t}=\frac{1}{\sqrt{2}}\left[\begin{array}{ll}
1 & 0 \\
0 & 0
\end{array}\right]\left[\begin{array}{l}
\cos \beta+i \sin \beta \\
\sin \beta-i \cos \beta
\end{array}\right]
$$

yield

$$
V_{1 R o t}=\frac{1}{\sqrt{2}}\left[\begin{array}{l}
\cos \beta-i \sin \beta \\
0
\end{array}\right]=\frac{1}{\sqrt{2}}\left[\begin{array}{l}
\cos (-\beta)+i \sin (-\beta) \\
0
\end{array}\right]
$$

and

$$
V_{2 R o t}=\frac{1}{\sqrt{2}}\left[\begin{array}{l}
\cos \beta+i \sin \beta \\
0
\end{array}\right]
$$

Eqs. (27) and (28) represent horizontally polarized light. The horizontal components are both complex, horizontal phases are $-\beta$ and $\beta$ respectively, again showing a relative phase of $2 \beta$. In conclusion: when for the polarization plane rotator a rotation angle of $\beta=\pi / 4$ is chosen, a phase step of $\pi / 2$ between the two counter-rotating circularly polarized beams is realized.

\section{SYSTEM DESCRIPTION}

A shearing speckle interferometer has been built ${ }^{8}$, implementing the phase stepping scheme based on the polarization plane rotator, as described above. The main components of the interferometer are the shearing section, the branch splitter and the combining unit (Fig. 4).

Randomly polarized light enters the system at the shearing section, which consists of a polarizing beam splitting cube, PBS1, two mirrors M1 and M2, and three quarter wave plates. PBS1 transmits horizontally polarized light towards fixed mirror M2 that reflects the light back towards PBS1. Between PBS1 and M2 a quarter wave plate oriented at $45^{\circ}$ takes care of changing the polarization direction from horizontal to vertical, causing the light now to be reflected to the output side of the shearing section. Similarly vertically polarized light is reflected towards mirror M1, the shearing mirror, that can be adjusted in order to obtain a slight change in direction of the beam. Again a quarter wave plate takes care of transforming the light, now from vertical to horizontal, causing the light to exit from PBS1 also at the output side. As a result two beams leave PBS1, one vertically polarized, the other one horizontally polarized and slightly sheared by the shearing mirror. A quarter wave plate at the exit of the PBS1, oriented at $45^{\circ}$, transforms the beams into a left- and a right-handed circularly polarized beam. This beam pair is split into two identical pairs by beam splitter BS1. One pair is reflected by a prism and $45^{\circ}$ mirror M3 towards the combining section, the other pair of circular beams is going straight through from branch splitter BS1 to the combiner. 


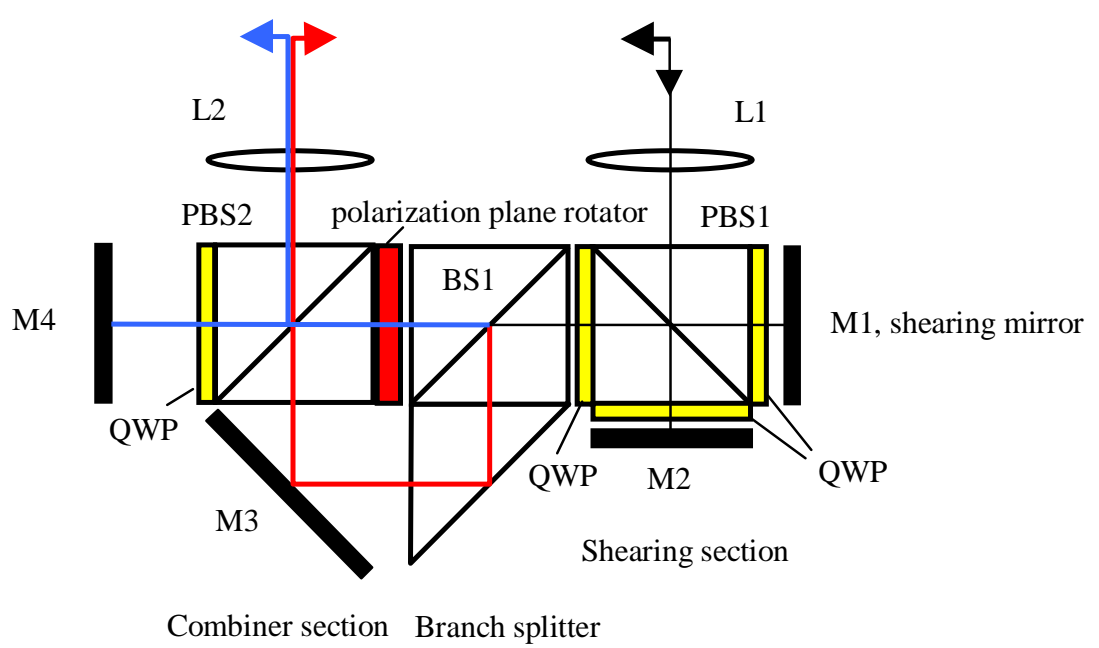

Figure 4: Single-camera two-channel polarization phase stepped shearing speckle interferometer.

The combiner section consists of polarizing beam splitter cube PBS2, mirror M4 and a quarter wave plate. PBS2 serves both as analyzer for both branches and as combining unit. The circular beams that are reflected by the prism and the $45^{\circ}$ mirror M3 travel through PBS2, after which the light of both beams is now linearly polarized in horizontal direction, causing the beams to interfere at the CCD camera. The other pair of circular beams is going straight through from branch splitter BS to the combiner. In this branch a polarizing plane rotator, rotating the circularly polarized beams over $\pi / 4$, effectuates a relative phase change between the beams of $\pi / 2$, as described above. This light travels through PBS2, after which it is horizontally polarized. It then reflects back from mirror M4 towards combiner PBS2, where it is reflected towards the CCD, after being converted to vertically polarized light by a quarter wave plate oriented at $45^{\circ}$, between combiner PBS2 and mirror M4.

Two images are now projected side by side onto the same CCD, where they are acquired simultaneously. The images are phase stepped by $\pi / 2$, and are processed according to the algorithms described in a previous section. Sub-pixel alignment of the two images is crucial, since speckle size is of the same order as pixel size. Precise alignment is achieved interactively by piezo-elements on mirror M3, using cross-correlation methods for real-time evaluation of the relative position of both speckle images ${ }^{10}$.

\section{EXPERIMENTAL}

The polarization plane rotator is positioned in a section of the geometrical optical system between two confocally placed relay lenses L1 and L2, together with all other components of the interferometer; light travels in parallel beams here, but at different angles. The configuration of the geometrical optical system is such that the maximum angle is not exceeding $3^{\circ}$, irrespective of the field angle at the entry side of the optical system. In order to test the properties of the polarization plane rotator some measurements have been carried out. The polarization state of linear light travelling through the polarization plane rotator was measured by measuring intensities in two directions: one in the direction with maximum intensity, referred to as main direction, the other perpendicular to it. The direction of maximum intensity appeared to be oriented at $45^{\circ}$ relative to the polarization direction of the incoming light, for all angles of incidence, within the measuring range. The direction perpendicular to the main direction showed intensities close to zero.

Results are shown in Fig. 5, where the ratio of light measured perpendicular to the main direction and in the main direction is plotted against the angle of incidence. It appears that light is only slightly elliptical at the edges of the actual range in the interferometer. The ratio between light measured perpendicular to the main direction and the light in the main direction is 0.0055 at an angle of incidence of $3^{\circ}$. 


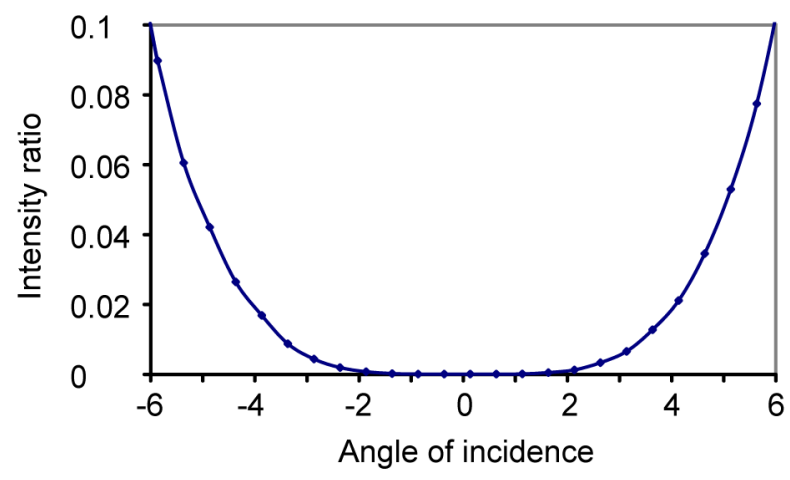

Figure 5: Transmission ratio for a $45^{\circ}$ polarization plane rotator, measured in the direction of maximum transmission and perpendicular to it, as a function of the angle of incidence. Ratio minimum / maximum is plotted.

\section{CONCLUSION}

A polarization phase stepping method has been presented for which a polarization plane rotator has been selected as the phase stepping component. With this component a phase step of $\pi / 2$ has been implemented, independent from the orientation of the rotator. Measurements on the properties of the rotator indicate that the useable range for its angle of incidence matches the existing range of the interferometer.

\section{ACKNOWLEDGMENTS}

This research was supported by the Technology Foundation STW, the Applied Science Division of NWO and the Technology Programme of the Ministry of Economic Affairs.

\section{REFERENCES}

1. K. Creath, "Phase-measurement interferometry techniques" in Progress in Optics, editor E. Wolf, Vol. 26, pp. 349393, Elsevier, 1988.

2. R. Crane, "Interference Phase measurement", Applied Optics, Vol. 8, pp. 538-542, 1969.

3. Gary E. Sommargren, "Up/down frequency shifter for optical heterodyne interferometry", J. Opt. Soc. Am. 65, pp. 960-961, 1975.

4. M. P. Kothiyal, "Polarization component phase shifters in phase shifting interferometry: error analysis", Opt. Acta, 33, pp. 787-793, 1986.

5. M. Kujawinska, "Spatial phase measurement methods," in Interferogram Analysis -Digital Fringe Pattern Measurement Techniques, D. W. Robinson and G. T. Reid, eds., Chap. 5, pp. 141-193, Institute of Physics, Bristol, U.K, 1993.

6. T. Bothe, J. Burke, H. Helmers, "Spatial phase shifting in ESPI: minimization of phase reconstruction errors", Applied Optics Vol. 36, No 22, pp. 5310-5316, 1997.

7. Qian Kemao, Miao Hong, Wu Xiaoping, "Real-time polarization phase shifting technique for dynamic deformation measurement", Optics and Lasers in Engineering, 31, pp. 289-295, 1999.

8. P.A.A.M. Somers and H. van Brug, "A single camera, dual image real-time-phase-stepped shearing speckle interferometer”, Fringe 2001, W. Osten, W. Jüptner, editors, pp. 573-580, Elsevier, Paris, 2001.

9. A.J.P. van Haasteren and H.J. Frankena, "Real-time displacement measurement using a multicamera phase-stepping speckle interferometer", Applied Optics Vol. 33, No. 19, pp. 4137-4142, 1994.

10. Peter A.A.M. Somers, Nandini Bhattacharya, "Maintaining sub-pixel alignment for a single camera two-bucket shearing speckle interferometer." Accepted for publication in a special issue of Journal of Optics A: Pure and Applied Optics, 2005. 
11. B. V. Dorrio and J. L. Fernandez, "Phase-evaluation methods in whole-field optical measurement techniques," Measurement Science \& Technology, Vol. 10, pp. R33-R55, 1999.

12. J.M. Huntley and H. Saldner, "Temporal phase-unwrapping algorithm for automated interferogram analysis", Applied Optics, Vol. 32, No. 17, pp. 3047-3052, 1993.

13. P A.A.M. Somers, H. van Brug, and J. J.M. Braat, "A two-bucket phase-stepped shearing speckle interferometer: why does it work," Speckle Metrology 2003 Proceedings, SPIE Vol. 4993, Kay Gastinger, Ole Jan Løkberg, Svein Winther, eds., pp. 181-188, SPIE, Bellingham, 2003.

14. Aldegonda L. Weijers, Hedser van Brug, Hans J. Frankena, "Polarization phase stepping with a Savart element", Applied Optics, Vol. 37, No. 22, pp. 5150-5155, 1998.

15. R.C. Jones, J. Opt. Soc. Am. 31, 488, 1941.

16. Frank L. Pedrotti, S.J, and Leno S. Pedrotti, Introduction to Optics, Prentice-Hall, Inc., Englewood Cliffs, 1987 , 1993. 